\title{
Determination of Trace Rhodamine B in Chili Oil by Deep Eutectic Solvent Extraction and an Ultra High-Performance Liquid Chromatograph Equipped with a Fluorescence Detector
}

\author{
Weida WANG, Yegang Du, Zeen XIAO, Yun LI, Bifang LI, and Guowu YANG ${ }^{\dagger}$ \\ Shenzhen Academy of Metrology and Quality Inspection, Shenzhen 518102, People's Republic of China
}

\begin{abstract}
Rhodamine B was forbidden in food by law because of its carcinogenic properties to humans. However, due to its low cost, it was often used to dope chili oil by some counterfeiters to improve its natural color. However, it was difficult to quantify rhodamine B in chili oil due to its complex substrates and high viscosity. In this study, deep eutectic solvents, comprised of choline chloride and ethylene glycol, were first used as an extraction medium to separate rhodamine B from chili oil.
\end{abstract}

Keywords Rhodamine B, deep eutectic solvent, chili oil

(Received November 3, 2016; Accepted January 12, 2017; Published June 10, 2017)

\section{Introduction}

Rhodamine B, with the chemical structure shown in Fig. 1, was used as an artificial synthetic xanthene dye, which was initially widely used in textiles and plastic industries as a colorant, and in biological chemistry as a fluorescent reagent. ${ }^{1-3}$ Rhodamine B was reported to have carcinogenic properties, neurotoxicity toxicity, and chronic toxicity to humans and animals. ${ }^{4,5}$ As a result of its harmful effects on human health, rhodamine B was identified as an illegal additive in food by the European Food Safety Authority. ${ }^{6}$ In the USA and China, the usage of rhodamine B in food was also forbidden. ${ }^{7}$ However, chili food was often adulterated with rhodamine B by some counterfeiters to improve the natural color due to its intense color and low cost. 6,8 Chili oil was a popular type of chili products in the market. Although many analytical methods were developed for the determination of rhodamine $\mathrm{B},{ }^{9-13}$ it was difficult to confirm and quantify when trace rhodamine B was mixed in chili oil owing to its high viscosity and complex natural colorants. Therefore, very few methods were reported to be suitable for testing chili oil samples. ${ }^{14-16}$ In a testing method reported by Prof. Zhang, rhodamine B and some natural pigments were separated from chili samples by methanol without selectivity. The other two methods suffered from complicated pretreatment and long time-consumption. Hence, the development of a particular, simple and cost-effective method suitable for chili oil samples remained a challenge.

Recently, deep eutectic solvents (DESs), which were developed by Abbott et al., attracted great attention due to promising properties, such as easy preparation, low cost, negligible vapor pressure, non-toxicity, and biodegradability. ${ }^{17-19}$ DESs composed of two or three inexpensive and safe components capable of self-association had low melting points compared to

W. W. and Y. D. contributed equally to this work.

${ }^{\dagger}$ To whom correspondence should be addressed.

E-mail: yangguowu126@126.com their constituting components. As a class of novel green solvents, DESs were applied to many fields, such as organic synthesis and compound separation. ${ }^{20-24}$ The current DESs were types of polar and water-miscible solvents, which should have potential ability to extract the analytes from oil samples. Qiu et al. separated phenolic compounds from model oil using DESs as extraction media. ${ }^{25}$

In this study, a particular, simple and cost-efficient method for the determination of trace rhodamine $\mathrm{B}$ in chili oil by deep eutectic solvent extraction and ultra-high-performance liquid chromatography (UPLC) equipped with a fluorescence detector was developed. The deep eutectic solvent (DES-1), ${ }^{26}$ comprised of choline chloride and ethylene glycol (mole ratio 1:3), was used as a green and inexpensive extractant to concentrate rhodamine B from chili oil samples. Interestingly, DES-1 showed good selectivity and efficient enrichment toward rhodamine B. Without any special cleanup procedures, there was no impurity interference with the determination of rhodamine B. To our knowledge, this was the first time that deep eutectic solvent extraction was applied to the analysis of rhodamine $\mathrm{B}$.

\section{Sample Preparation and Analytical Procedures}

A portion of chili oil (1.0 g) was accurately weighed into a<smiles>CCN(CC)c1ccc2c(-c3ccccc3C(=O)O)c3ccc(=[N+](CC)CC)cc-3oc2c1</smiles>

Fig. 1 Chemical structure of rhodamine B. 


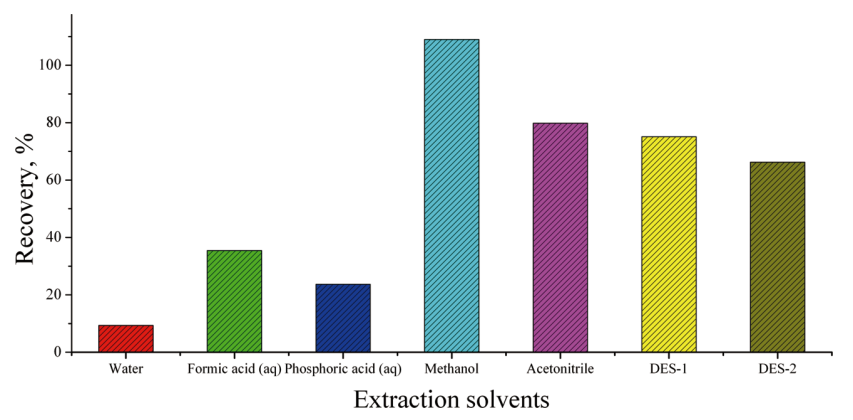

Fig. 2 Extraction efficiency of rhodamine $\mathrm{B}$ with pure water, formic acid/ammonium formate buffer $(0.01 \mathrm{M}, \mathrm{pH} 3)$, phosphoric acid/ potassium dihydrogen phosphate $(0.01 \mathrm{M}, \mathrm{pH} 3)$, methanol, acetonitrile, DES-1 and DES-2 from spiked samples $\left(5 \mu \mathrm{g} \mathrm{kg}^{-1}\right)$.

$15 \mathrm{~mL}$ centrifuge tube, followed by the addition of DES-1 $(1.0 \mathrm{~mL})$ and $n$-hexane $(5 \mathrm{~mL})$. This mixture was shaken under vortex movement for $1 \mathrm{~min}$, and then centrifuged at $7800 \mathrm{rpm}$ for $3 \mathrm{~min}$ to accelerate phase separation. The upper phase was removed using a plastic pipette. The residue was washed again with $n$-hexane $(3 \mathrm{~mL})$, and then diluted to a total volume of $5 \mathrm{~mL}$ with methanol. The final solution was filtered through a $0.22-\mu \mathrm{m}$ membrane for UPLC analysis.

UPLC analysis conditions: The chromatographic conditions were also investigated. Finally, water (solvent A) and methanol (solvent B) were used as the mobile phase, and a gradient elution method was employed in order to obtain a suitable retention time and the best signal to noise ratio. The linear gradient conditions were as follows: $0.0 \mathrm{~min}, 30 \% \mathrm{~B} ; 3.0 \mathrm{~min}$, $90 \% \mathrm{~B} ; 5.0 \mathrm{~min}, 90 \% \mathrm{~B} ; 6.0 \mathrm{~min}, 30 \% \mathrm{~B} ; 7.0 \mathrm{~min}, 30 \% \mathrm{~B}$. The flow rate was $0.3 \mathrm{~mL} / \mathrm{min}$. The column oven temperature was set to $30^{\circ} \mathrm{C}$ and the injection volume was $3 \mu \mathrm{L}$. The excitation and emission wavelength were set at 550 and $580 \mathrm{~nm}$, respectively, for fluorescence data collection.

\section{Results and Discussion}

In the established method, rhodamine B was extracted from chili oil with DES-1 comprised of choline chloride and ethylene glycol (mole ratio 1:3). In order to stress the extraction behavior of DES-1 for rhodamine B in chili oil, an equal volume of water as the extraction medium was used for a control experiment in consideration of the aqueous solubility of rhodamine B. Compared with the approximately $75 \%$ recovery from DES -1 , the recovery from water was less than $10 \%$ (shown in Fig. 2). Taking into account the structure of rhodamine B, acidic buffer solutions may provide higher recovery than water. As expected, both formic acid/ammonium formate buffer and phosphoric acid/potassium dihydrogen phosphate buffer gave higher recovery than water. However, the recovery from acidic buffer solutions was still far less than that from DES-1. As a traditional organic solvent, methanol dramatically improved the extraction efficiency of rhodamine B from chili oil samples. Although methanol was previously used quite often as an extraction solvent for rhodamine B, some natural pigments were separated from chili samples together with rhodamine B as shown in Fig. 3. Acetonitrile, if served as an extractant, was highly toxic to human. Moreover, the influence of mole ratio of choline chloride and ethylene glycol on the recovery in the proposed extraction method was investigated. According to previously reported research, the deep eutectic solvent (DES-2) comprised of choline chloride and ethylene glycol (mole ratio 1:2) ${ }^{27,28}$ was

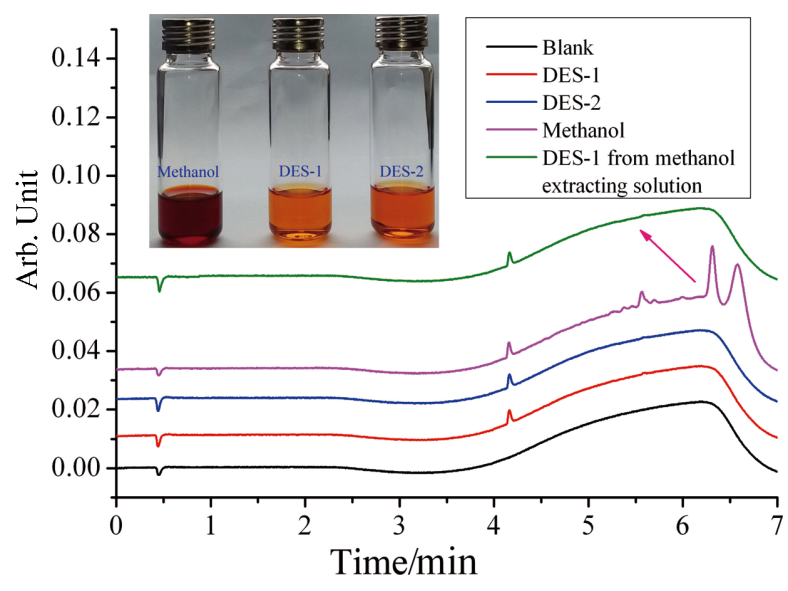

Fig. 3 UV-Vis chromatograms of extracting solutions at $550 \mathrm{~nm}$ with methanol, DES-1 and DES-2. The insert shows a simple colorimetric assay.

also applied to the separation of rhodamine B from chili oil. As the results demonstrated, the optimum mole ratio of choline chloride and ethylene glycol was 1:3. This may be ascribed to the viscosity of the DESs which varied in the sequence DES-1 $<$ DES-2. ${ }^{25}$ In spite of the different extraction efficiency, the deep eutectic solvents DES-1 and DES-2 showed better selectivity for rhodamine B than some natural pigments, as displayed in UV-Vis chromatograms at $550 \mathrm{~nm}$ (presented in Fig. 3). To emphasize the selectivity behavior of DES-1 compared with methanol, the methanol extracting solution was extracted with DES-1 after a nitrogen flush treatment. The other peaks, except for rhodamine $\mathrm{B}(4 \mathrm{~min})$ on the chromatogram, disappeared, as revealed in Fig. 3 (the top line), which clearly demonstrated high selectivity of DES-1 for rhodamine B. Although our method is based on fluorescence detection, UV-Vis detection at $550 \mathrm{~nm}$ was used to evaluate the selectivity of the extraction solvents for two reasons. First, the types of pigments showing absorption properties in chili oil were much more than that of pigments showing fluorescence emission properties. Second, one pigment should have absorption at $550 \mathrm{~nm}$ if it could be detected by our proposed fluorescence method. Therefore, the data of absorbance could fully stress the selectivity of DES-1 for rhodamine B.

The optimization of extraction conditions were studied, and the details was depicted in Figs. S1 and S2 (Supporting Information). The extraction time (1 min) required for extraction equilibrium in this proposed method was much less than that of previously reported methods. ${ }^{14-16}$

\section{Method validation}

A calibration curve was constructed using six working standard solutions in the range of $1.00-50.0 \mu \mathrm{g} / \mathrm{L}$ to examine the linearity. The obtained regression equation and correlation coefficient were $y=2.48 \times 10^{4} x+2.26 \times 10^{2}, \quad R 2=0.9996$. The precision and accuracy of the analytical method were estimated through the relative standard deviation (RSD, \%) and recovery (\%), respectively, in the recovery experiments. A blank value was determined prior to the recovery study. Spiked samples with three additive levels $(10,50$, and $200 \mu \mathrm{g} / \mathrm{kg})$ were obtained by delivering $0.2 \mathrm{~mL}$ of standard solutions $(50,250$, $1000 \mu \mathrm{g} / \mathrm{L}$ ) to $1 \mathrm{~g}$ of chili oil. The parallel experiments for each level involved 6 samples. The limits of detection (LOD) and quantification (LOQ) were determined by the signal-to-noise 

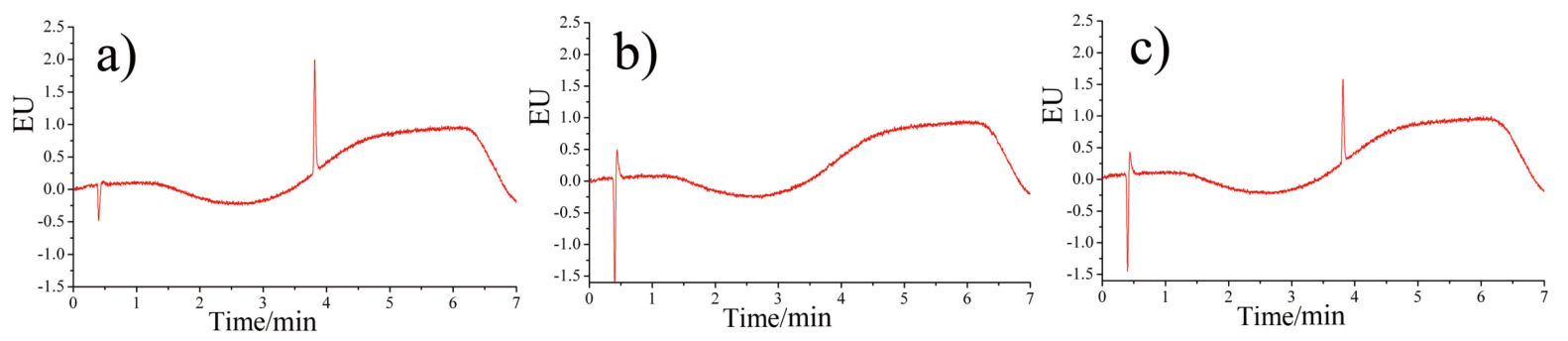

Fig. 4 Fluorescence chromatograms of the rhodamine B standard solution (a, $\left.2.00 \mu \mathrm{g} \mathrm{L}^{-1}\right)$, the real sample marked with 1 (b) and the spiked sample (c, $10 \mu \mathrm{g} \mathrm{kg}^{-1}$ ).

$S / N=3$ and 10 ratios of the minimum peak in the standard solutions, which were calculated to be 1.67 and $5 \mu \mathrm{g} / \mathrm{kg}$, respectively. The precision and accuracy results are given in Table S1.

\section{Real sample application}

For estimating a practical application value of this method, eight chili oil samples marked with 1 to 8 that were purchased from local markets were used as analysis objects. Figure 4 shows chromatograms of the real sample marked with 1 and a spiked sample. The test results are demonstrated in Table S2, which lists the concentrations of rhodamine $\mathrm{B}$ in real chili oil samples and the recovery of the spiked real samples (the final concentrations was $10 \mu \mathrm{g} / \mathrm{kg}$ ). Quantification was performed by the standard calibration curve. The spiked recovery values of the analytes ranged from 56.2 to $73.9 \%$. This developed method appeared to be an appropriate technique for analyzing rhodamine $\mathrm{B}$ at trace-level in actual samples.

\section{Conclusions}

In summary, a deep eutectic solvent (DES-1) comprised of choline chloride and ethylene glycol (mole ratio 1:3) could selectively separate rhodamine B from chili oil. It was the first time a deep eutectic solvent (DES) was used as a novel extraction medium for the determination of rhodamine B. The established method using DES-1 as the extraction agent was a sensitive, convenient and simple analytical technique for determining rhodamine B in real chili oil samples, and had great significance for market supervision. Importantly, this method had potential application in rapid qualitative detection of rhodamine B in chili oil. Moreover, this study had explored the potential capacity of deep eutectic solvents in depth for the characterization of chemical contaminants in food.

\section{Supporting Information}

This material is available free of charge on the Web at http:// www.jsac.or.jp/analsci/.

\section{References}

1. T. L. Chiang, Y. C. Wang, and W. H. Ding, J. Chin. Chem. Soc., 2012, 59, 515.

2. M. Yasuda and T. Akimoto, Anal. Sci., 2015, 31, 139.

3. T. H. A. Hasanin, Y. Tsunemine, S. Tsukahara, Y. Okamoto, and T. Fujiwara, Anal. Sci., 2011, 27, 297.
4. H. H. Gan, G. K. Zhang, Y. L. Zhang, and Y. D. Guo, Desalin. Water Treat., 2012, 45, 112.

5. R. Jain, M. Mathur, S. Sikarwar, and A. Mittalet, J. Environ. Manage., 2007, 85, 956.

6. S. Lin, W. L. J. Hasi, X. Lin, S. Q. G. W. Han, X. T. Lou, F. Yang, D. Y. Lin, and Z. W. Lu, Anal. Methods, 2015, 7, 5289.

7. J. J. Shi and L. G. Chen, Anal. Methods, 2014, 6, 8627.

8. J. Sun, T. Gan, Y. Li, Z. X. Shi, and Y. M. Liu, J. Electroanal. Chem., 2014, 724, 87.

9. E. Ranjbari, M. R. Hadjmohammadi, F. Kiekens, and K. D. Wael, Anal. Chem., 2015, 87, 7894.

10. M. Soylak, Y. E. Unsal, E. Yilmaz, and M. Tuzen, Food Chem. Toxicol., 2011, 49, 1796.

11. C. Tatebe, X. N. Zhong, T. Ohtsuki, H. Kubota, K. Sato, and H. Akiyama, Food Sci. Nutr., 2014, 2, 547.

12. N. Pourreza, S. Rastegarzadeh, and A. Larki, Talanta, 2008, 77, 733.

13. M. Taziki, F. Shemirani, and B. Majidi, Sep. Purif. Technol., 2012, 97, 216.

14. P. Qi, Z. H. Lin, J. X. Li, C. L. Wang, W. W. Meng, H. Hong, and X. W. Zhang, Food Chem., 2014, 164, 98.

15. M. Oplatowska and C. T. Elliott, Analyst, 2011, 136, 2403.

16. P. Botek and J. Poustka, Czech J. Food Sci., 2007, 25, 17.

17. A. P. Abbott, G. Capper, D. L. Davies, R. K. Rasheed, and V. Tambyrajah, Chem. Commun., 2003, 70.

18. J. A. Hammons, J. Ustarroz, T. Muselle, A. A. J. Torriero, H. Terryn, K. Suthar, and J. Ilavsky, J. Phys. Chem. C, 2016, 120, 1534.

19. P. Liu, J. W. Hao, L. P. Mo, and Z. H. Zhang, RSC Adv., 2015, 5, 48675.

20. S. B. Phadtare and G. S. Shankarling, Green Chem., 2010 , 12, 458.

21. R. Martínez, L. Berbegal, G. Guillena, and D. J. Ramón, Green Chem., 2016, 18, 1724.

22. Q. Zeng, Y. Z. Wang, Y. H. Huang, X. Q. Ding, J. Chen, and K. J. Xu, Analyst, 2014, 139, 2565.

23. K. Pang, Y. C. Hou, W. Z. Wu, W. J. Guo, W. Peng, and K. N. Marsh, Green Chem., 2012, 14, 2398.

24. A. P. Abbott, P. M. Cullis, M. J. Gibson, R. C. Harris, and E. Raven, Green Chem., 2007, 9, 868.

25. T. N. Gu, M. L. Zhang, T. Tan, J. Chen, Z. Li, Q. H. Zhang, and H. D. Qiu, Chem. Commun., 2014, 50, 11749.

26. K. Shahbaz, S. Baroutian, F. S. Mjalli, M. A. Hashim, and I. M. AlNashef, Thermochim. Acta, 2012, 527, 59.

27. K. Shahbaz, F. S. Mjalli, M. A. Hashim, and I. M. AlNashef, Thermochim. Acta, 2011, 515, 67.

28. C. D'Agostino, R. C. Harris, A. P. Abbott, L. F. Gladden, and M. D. Mantle, Phys. Chem. Chem. Phys., 2011, 13, 21383. 